\title{
A 10-Gb/s 1024-Way-Split 100-km Long-Reach Optical-Access Network
}

\author{
Darren P. Shea and John E. Mitchell, Member, IEEE
}

\begin{abstract}
Optical-access networks have been developed to remove the access-network bandwidth bottleneck. However, the current solutions do not adequately address the network economics to provide a truly cost-effective solution. Long-reach optical-access networks introduce a cost-effective solution by connecting the customer directly to the core network, bypassing the metro network, and, hence, removing significant cost. This paper charts the design and development of a 1024-way-split 100-km 10-Gb/s symmetrical network, which experimentally proves the feasibility of long-reach optical-access networks for both the upstream and downstream transmission.
\end{abstract}

Index Terms-Long reach, next-generation access, optical access, $10 \mathrm{~Gb} / \mathrm{s}$.

\section{INTRODUCTION}

$\mathbf{L}$ ONG-REACH optical-access networks represent the next step in the evolution of optical-access networks that will deliver future high-bandwidth services. Optical technologies have enabled a massive explosion in the bandwidth that is achievable in core networks. Technologies such as optical amplifiers, dense wavelength-division multiplexing, forward error correction (FEC), etc., have permitted a reduction in opticaltransmission penalties and have increased the capacities of fibers. Unfortunately, most customers do not have access to the comparatively unlimited core bandwidth as the majority of access network connections are based on copper lines. Copperbased technologies have been developed that have increased the access bandwidth to $\sim 2 \mathrm{Mb} / \mathrm{s}$ and greater depending on the length and quality of the copper lines. However, with future services forecast to require much greater bandwidths, a high bandwidth alternative must be found.

Passive optical networks (PON) are a promising option for a cost-effective solution to this bandwidth problem. A single fiber extends from the local exchange on the access/metro network edge to a location close to the customer premises. The fiber is then passively split into a number of individual fibers, which form the direct link to the customer premises. The first and second generation of PONs have been standardized [1], [2] and are currently being deployed in some areas as interest in opticalaccess technologies gather speed.

Manuscript received March 22, 2006; revised December 1, 2006. This work was supported in part by the EPSRC and BT.

The authors are with the Department of Electronic and Electrical Engineering, University College London, Torrington Place, London WC1E 7JE, U.K. (e-mail: dshea@ee.ucl.ac.uk; jmitchel@ee.ucl.ac.uk).

Color versions of one or more of the figures in this paper are available online at http://ieeexplore.ieee.org.

Digital Object Identifier 10.1109/JLT.2006.889667
Based on the principles of PON, long-reach optical-access networks provide further cost savings by extending the physical reach of the network to the core network. This removes the need for additional equipment to connect the access network to the core. Second, the cost of the shared equipment in the network is reduced by increasing the split size. Long-reach optical-access networks would provide a cost-effective solution for operators to install optical-access technology.

In this paper, we present an overview of our work on longreach optical-access networks. In Section II, the economic and technical justification of a 1024-way-split 100-km 10-Gb/s long-reach optical-access networks is presented. The network is designed from the perspective of a network operator, recognizing the economic and physical constraints faced when installing new technology into the access network. Section III examines the key parameters of both the upstream and downstream transmission paths using analytical modeling and simulation for continuous-mode operation. Physical proof of the feasibility of a long-reach access network is presented in Section IV, which details the construction and experimental analysis of a continuous-mode laboratory demonstrator. Section $\mathrm{V}$ provides a brief summary of the results and a conclusion.

\section{Network ARChitecture}

Introducing fiber into the access network will remove the bandwidth bottle-neck. A major disadvantage of a fiber-optic solution is the expense of installation. In the U.K., the current BT access network serves 23 million customers over 34 million lines (including spare capacity) [3]; replacing each line would not be easy logistically or financially. The amount of fiber required to replicate the current copper access network makes a point-to-point optical-access network expensive. An alternate economic network infrastructure must be developed.

\section{A. Economic Drivers}

PON presents a feasible alternative to point-to-point networks, through a tree-and-branch-type structure. A single fiber extends from the local-exchange site, which is split through a passive optical splitter into individual fibers. The passive optical splitter is placed at a location close to the customer premises so that each customer fiber is of limited length. The amount of fiber is reduced in comparison to a point-to-point network, reducing the cost per customer, as each customer no longer requires a dedicated fiber from the local exchange to the premises. Current PONs offer a point-to-multipoint fiber 
network, but operators have been slow to introduce them into their networks due to the installation costs. Hence, to encourage operators to install fiber in the access network, optical-access networks must be made more economic.

Cost savings can be achieved by increasing the split size, which increases the number of customers across which the cost of the shared equipment is spread. The largest and highest capacity PON currently available is the GPON, standardized by the International Telecommunication Union (ITU) [2], which is capable of sharing $2.5 \mathrm{~Gb} / \mathrm{s}$ between a maximum of 64 customers over $20 \mathrm{~km}$. In GPON, the shared equipment cost is still significant on a cost-per-customer basis as it is only shared over a maximum of 64 users. However, in point-tomultipoint networks, the total capacity of the network is shared between all users. Increasing the number of users without a corresponding increase in the data rate results in a reduction in the bandwidth each customer receives.

Increasing the network data rate to $10 \mathrm{~Gb} / \mathrm{s}$ would counteract this issue but requires a more expensive high-speed transmitter to be used in each optical network unit (ONU). The cost of the ONU can be reduced by making it as simple as possible through the addition of equipment in the shared section of the network to improve the network performance. Adding equipment to the shared section of the network is economic as the cost increase is minimal due to the large split size. Moving equipment deeper into the network enables cost sharing; each customer is only required to pay a fraction of the cost, e.g., the optical line terminal (OLT) situated at the head end of the network, could be shared by 1024 users; therefore, each user only contributes 1/1024th of the total OLT cost. Shared equipment can, therefore, be more expensive without a dramatic increase in the overall network cost per user. This enables expensive equipment such as optical amplifiers, dispersioncompensating techniques, etc., to be introduced in the shared section of the network with minimal effect on the cost per user.

An additional capacity increase is also gained from the statistical multiplexing provided by increasing the number of user to 1024. The capacity available to each customer will depend on the utilization of the network. As not every user shall be active at all times, the network capacity can be shared dynamically between the active users.

Increasing the range of the network can provide further benefits to the operator. Telecoms networks are extremely complex and usually consist of a number of network tiers [4]. Simplification can be introduced through bypassing the metro network by extending the reach of the access network to $100 \mathrm{~km}$ to enable a direct connection to the core network. Total network costs are reduced as there is no longer a requirement for additional equipment, such as synchronous digital hierarchy (SDH) rings to connect the access and core networks. Eventually, as longreach optical-access networks deployment increases, services can migrate to the new platform, making the metro network and its equipment redundant. Removal of the metro equipment would free space in local-exchange sites, possibly allowing smaller sites to be removed, providing real-estate savings. The simplification provided through the single network solution, coupled with the reliability of fiber, significantly reduces the maintenance and operating costs of the network.
In summary, network operators have been reluctant to implement optical-access networks in the past due to the unattractive financial aspects, namely the large capital expenditure associated with the installation of the large amount of fiber required. It is possible to make optical-access networks more attractive and more feasible economically through careful design of the network. A point-to-multipoint architecture reduces the fiber requirements; increasing the split size reduces the cost of shared equipment; using expensive technology into the shared section of the network reduces cost of the ONU; and extending the reach simplifies the overall network and provides cost savings through equipment/building redundancy and operational savings. Therefore, an access network with a reach of $100 \mathrm{~km}$, 1024-way split at $10 \mathrm{~Gb} / \mathrm{s}$ (symmetric) is proposed to fit all of the above requirements.

\section{B. Technical}

The physical layer of a standard nonamplified PON can be defined by the available optical power budget, which consists of the sum of optical powers and losses. The GPON optical power budget is designed to support a maximum split size of 64 with $20-\mathrm{km}$ reach operating at $2.5 \mathrm{~Gb} / \mathrm{s}$ and, therefore, is not large enough to cope with the greater split size, reach, and data rate of the proposed system. A common method of increasing optical power budgets in core and subsea networks is to use optical amplifiers. Optical amplifiers are a feasible option as the prices have been eroded through volume production and the technology has been proved to be suitable through their use in other systems.

In the upstream direction (from customer to central office), the optical signal transmitted from the ONU transmitter is immediately faced with a large amount of attenuation due to the split loss in the distribution section. A 1024-way split is made up of a cascade of two $\mathrm{N}: 16(2 \times 14 \mathrm{~dB}=28 \mathrm{~dB}$ [5]) and one $\mathrm{N}: 4(7.3 \mathrm{~dB}$ [5]) splitter plus 10-km fiber loss $(0.35 \mathrm{~dB} / \mathrm{km} \times 10 \mathrm{~km}=3.5 \mathrm{~dB})$. Also included are $0.5-$ and $1-\mathrm{dB}$ loss due to flexibility points at the customer premises and the local-exchange building, respectively. This results in a total distribution section loss of $40.3 \mathrm{~dB}$. A conservative fiber loss coefficient of $0.35 \mathrm{~dB} / \mathrm{km}$ is assumed as a worstcase loss for installed fiber, which may be reused. Due to economic constraints in the upstream transmission path, a lowcost transmitter must be used in the ONU at the customer premises. Generally, low-cost transmitters have a low-output power, which means a very weak signal appears at the input to the local-exchange site due to the distribution-section loss. Placing an optical amplifier at this point would increase the power of the attenuated optical signal.

The local-exchange site is a good choice due to the existing availability of electrical power; hence, no new power cabling would need to be installed and the optical amplifier is placed after the split, in the shared part of the network, so that the cost is shared among all users. The latter point conforms to the principle of simplifying the ONU by adding equipment in the shared section to make the system more cost-effective per user. A rough calculation suggests that a single amplifier at the local-exchange site after the split will not be enough for good 


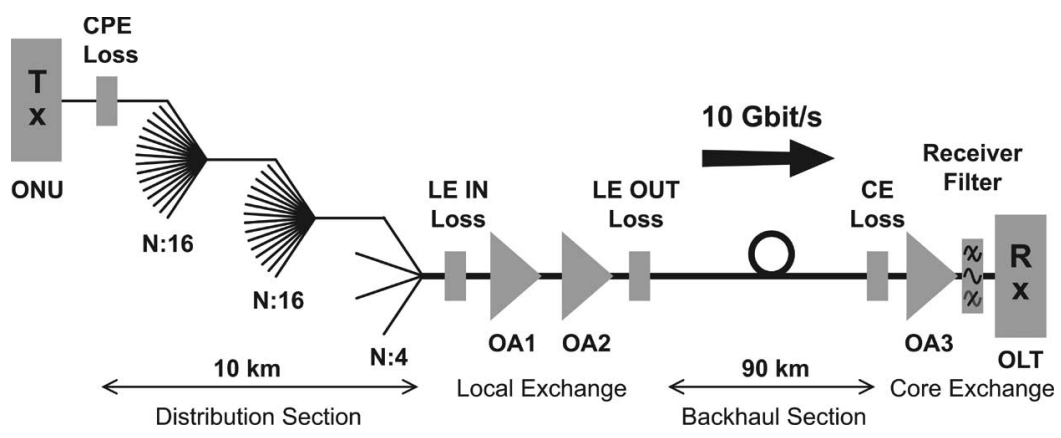

Fig. 1. Proposed upstream architecture for long-reach optical-access networks.

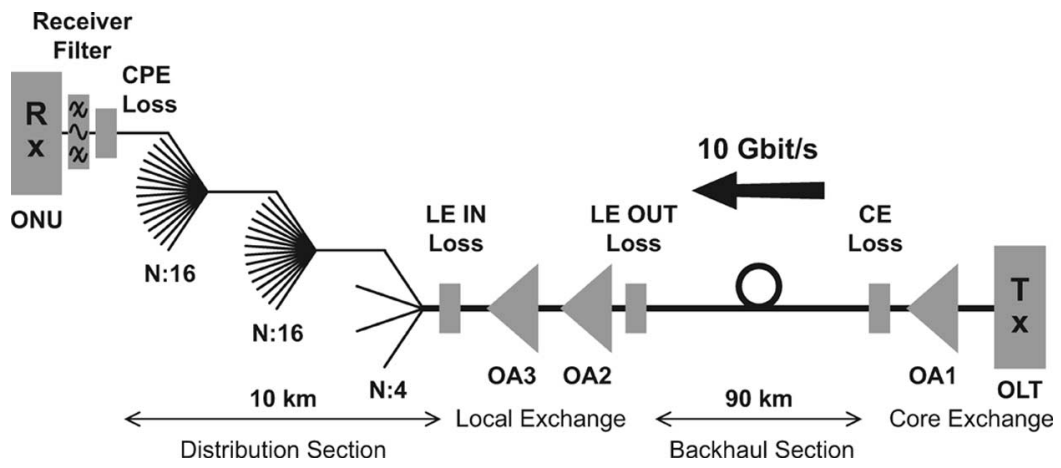

Fig. 2. Proposed downstream architecture for long-reach optical-access networks.

system performance, as the input signal to the optical amplifier will be extremely small-in the region of $-40 \mathrm{dBm}$. Assuming the optical amplifier has a gain of $25 \mathrm{~dB}$, the maximum output power from the optical amplifier is $-15 \mathrm{dBm}$. Attenuation in the backhaul section is $34 \mathrm{~dB}$ due to the $90 \mathrm{~km}$ of fiber plus additional flexibility point losses. At the receiver, the signal would therefore be $-49 \mathrm{dBm}$, well below the typical $-20 \mathrm{dBm}$ $\left(\mathrm{BER}=10^{-10}\right)$ sensitivity of a practical $10-\mathrm{Gb} / \mathrm{s}$ receiver. Adding another optical amplifier at the local-exchange site would be feasible as the cost would be shared between all customers and electrical power is available. However, even with an additional $25 \mathrm{~dB}$ of gain, the signal power at the receiver would be at best $-24 \mathrm{dBm}$, which is below the receiver sensitivity of a practical receiver. A solution is to increase the sensitivity of the receiver by adding an optical amplifier immediately before the receiver to form an optically preamplified receiver. Such devices operating at $10 \mathrm{~Gb} / \mathrm{s}$ have been reported with sensitivities of $-37.2 \mathrm{dBm}$ at $\mathrm{BER}=10^{-9}$ [6]. Therefore, using two amplifiers at the local-exchange site with an optically preamplified receiver would potentially constitute a feasible system. Upstream and downstream architectures for long-reach optical-access networks are given in Figs. 1 and 2, respectively. The downstream architecture is symmetrical with the upstream architecture, with optical amplifiers located at the local-exchange site and the core exchange.

It must be noted that extending the reach and the inclusion of optical amplifiers introduce impairments [i.e., dispersion and amplified spontaneous emission (ASE), respectively] that are not present in standard PONs. The following section seeks to study and reduce these impairments in order to optimize the system performance.

\section{Key PARAMETERS}

An in-depth analysis of the upstream and the downstream transmission paths was performed using an analytical model [7]. The analytical model studied the upstream and downstream transmission paths in a loss-only environment to identify the parameters which are of critical importance to ensure the target system performance of $\mathrm{BER}=10^{-10}(Q=16 \mathrm{~dB})$ can be achieved. Further analysis of the key parameters identified by the analytical modeling was performed using a commercial simulation package called OptSim, which allowed dispersive and nonlinear effects to be studied. Parameters of long-reach optical-access networks used in both the analytical modeling and simulations are given in Table I. Standard single-mode fiber is used in both transmission directions.

\section{A. Upstream Parameters}

In this section, we consider the component values required for optimum performance in the upstream. Starting with the transmitter, we first identify the frequency-chirping factor required for optimum transmission. Second, the ASE penalty due to each optical amplifier (OA) is calculated analytically before using OptSim simulations to determine the required saturation power for each OA. In each case, the relevant component values from previous section are used.

1) Transmitter: As the ONU transmitter is a cost-critical component, it is important to set $P_{\mathrm{Tx}}$ to be as low as possible to save cost. Simulations of the upstream architecture, given in Fig. 1, were performed to study how the transmitter frequencychirping parameter $\alpha_{c}$ can be tuned to reduce the effect of 
TABLE I

LONG-REACH OPTICAL-ACCESS NETWORK PARAMETERS

\begin{tabular}{llrl}
\hline \hline Symbol & \multicolumn{1}{c}{ Quantity } & Value & Unit \\
\hline$\gamma$ & wavelength & 1550 & $\mathrm{~nm}$ \\
$\mathrm{r}$ & extinction ratio & 10 & $\mathrm{~dB}$ \\
$\mathrm{~L}_{\mathrm{ONU}}$ & ONU connector loss & 0.5 & $\mathrm{~dB}$ \\
$\alpha$ & fibre loss coefficient & 0.35 & $\mathrm{~dB} / \mathrm{km}$ \\
Length & distribution section length & 10 & $\mathrm{~km}$ \\
$1: 1024$ & 1024 way split loss & 35.3 & $\mathrm{~dB}$ \\
$\mathrm{~L}_{\mathrm{RCU}}$ IN & local exchange loss IN & 1 & $\mathrm{~dB}$ \\
$\mathrm{G}_{\mathrm{OA}}$ & optical amplifier gain & 25 & $\mathrm{~dB}$ \\
$\mathrm{~F}^{\prime}$ OA & optical amplifier noise figure & 5 & $\mathrm{~dB}$ \\
$\mathrm{~L}_{\mathrm{RCU} \text { OUT }}$ & local exchange loss OUT & 1 & $\mathrm{~dB}$ \\
Length $_{\mathrm{BH}}$ & backhaul section length & 90 & $\mathrm{~km}$ \\
$\mathrm{~L}_{\mathrm{TE}}$ & core exchange loss & 1.5 & $\mathrm{~dB}$ \\
Rx $_{\text {SENS }}$ & receiver sensitivity (@ 10-10) & -20 & $\mathrm{dBm}$ \\
$\mathrm{B}_{\mathrm{e}}$ & receiver electrical bandwidth & 7.5 & $\mathrm{GHz}$ \\
\hline \hline
\end{tabular}

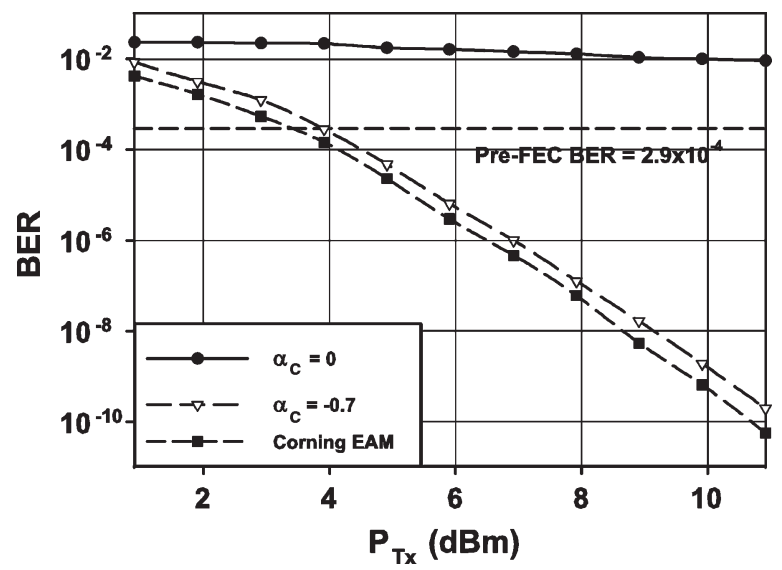

Fig. 3. External modulation-transmitter simulations (OptSim) for the upstream transmission path including dispersion. Upstream performance is simulated when frequency chirping, $\alpha_{C}$ is set to 0 and -0.7 , and when we use a Corning device. Extinction ratio $=10 \mathrm{~dB}$ and receiver filter optical bandwidth of $20 \mathrm{~nm}$ for all simulations. All other parameters as in Table I.

dispersion at $1550 \mathrm{~nm}$ in nondispersion-shifted fiber. Results of the OptSim simulations are given in Fig. 3. Direct modulation simulations failed to achieve the minimum performance threshold as a result of pulse broadening due to $\alpha_{c}=3.5$ [8]. Hence, direct modulation was discounted and is not included in Fig. 3.

FEC is increasingly being used as a method of increasing performance in optical-transmission systems. ITU standard G.975 [9] defines FEC using a Reed-Solomon code $[\operatorname{RS}(255,239)$ for submarine systems], as used in the G.984 GPON standards. The additional FEC overhead is calculated by dividing the codeword length by the block size [255/239 for RS(255, 239)], resulting in $\sim 7 \%$ for $\operatorname{RS}(255,239)$. Therefore, the data rate must be increased to $10.7 \mathrm{~Gb} / \mathrm{s}$; otherwise, the amount of data transmitted is reduced by $7 \%$. The system performance is required to be $\mathrm{BER}=10^{-10}(Q=16 \mathrm{~dB})$; hence, a useful value would be pre-FEC BER target required to achieve a post-FEC $\mathrm{BER}=10^{-10}$. An approximate pre-FEC target of $\mathrm{BER}_{\mathrm{IN}}=$
TABLE II

PRE-FEC BER FOR BER $=10^{-10}$ IN PRACTICAL SYSTEMS

\begin{tabular}{ll}
\hline \hline System & Pre-FEC BER \\
\hline 1 & $2.8 \times 10^{-4}[12]$ \\
2 & $2.7 \times 10^{-4}[13]$ \\
3 & $5.0 \times 10^{-5}[14]$ \\
4 & $5.5 \times 10^{-4}[15]$ \\
Average & $2.9 \times 10^{-4}$ \\
\hline \hline
\end{tabular}

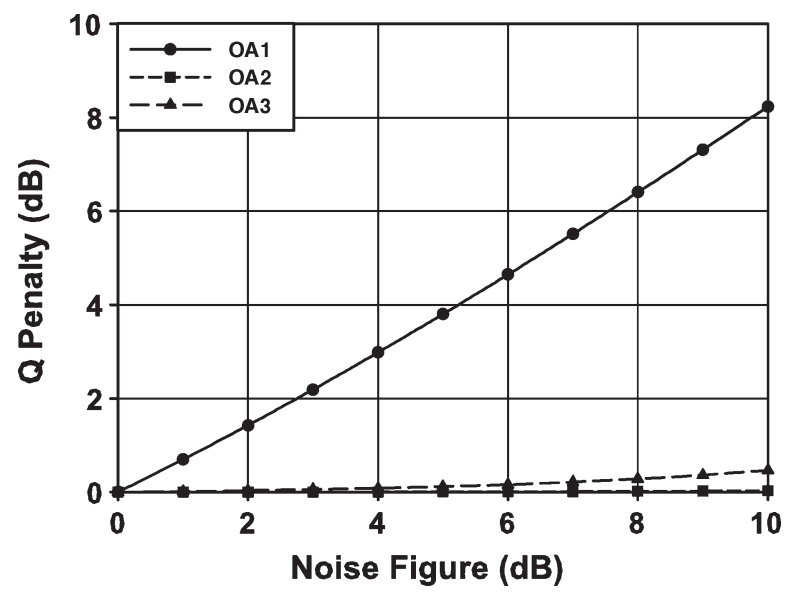

Fig. 4. Penalty placed on long-reach optical-access networks performance by $\mathrm{OA} 1, \mathrm{OA} 2$, and OA3 with receiver filter optical bandwidth $=20 \mathrm{~nm}$ calculated using the analytical model.

$2.9 \times 10^{-4}(Q=10.7 \mathrm{~dB})$ was obtained by studying previous systems, which have used $\operatorname{RS}(255,239)$ FEC. A summary is given in Table II.

Referring to Fig. 3, setting $\alpha_{c}=-0.7$ [10] improves system performance over $\alpha_{c}=0$ so that the minimum $P_{\mathrm{Tx}}$ to achieve the pre-FEC BER $=2.9 \times 10^{-4}$ is $4 \mathrm{dBm}$. Further performance increases can be gained by using an electro-absorption modulator (EAM) device, where $\alpha_{c}$ varies depending on the electrical-drive conditions [8]. The frequency-chirping factor of an EAM manufactured by Corning varies from $\alpha_{c 1}=0.5$ in its on state to $\alpha_{c 0}=-2$ in its off state $\left(\alpha_{c 1}=\mathrm{ON}, \alpha_{c 0}=\mathrm{OFF}\right)$ [11]. Simulations of the Corning device are included in Fig. 3, which demonstrates that the best performance can be achieved with $\alpha_{c}<-0.7$. Further simulations revealed that the optimum frequency chirping for the long-reach optical-access network is $\alpha_{c 1}=-1.4$ and $\alpha_{c 0}=-1.2$.

2) ASE Penalty: The beneficial effects of optical amplification are offset by the generation of ASE noise as a byproduct of the optical-amplification mechanism. To assess the detrimental effects of ASE on this system, the noise figure of each optical amplifier was varied in the analytical model, calculating the penalty induced by comparing the system performance, in terms of $Q$ in decibels, to the ideal case where $F^{\prime}=0 \mathrm{~dB}$ for the amplifier in question. For OA1, the noise figures of OA2 and $\mathrm{OA} 3$ were set to 6 and $5 \mathrm{~dB}$, which are typical values for booster and preamplifier values, respectively. When the penalties for $\mathrm{OA} 2$ and $\mathrm{OA} 3$ are calculated, the noise figure of OA1 is set at 5 dB. Fig. 4 shows the overall system penalty calculated for 


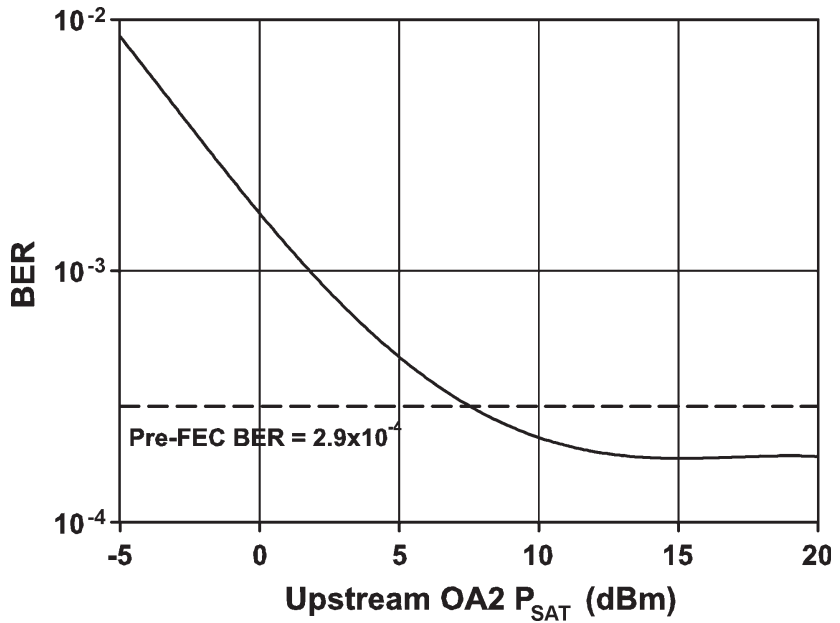

Fig. 5. Upstream system performance when varying $P_{\mathrm{SAT}}$ from -5 to $20 \mathrm{dBm}$ in OA2 for a 1024-way-split $100-\mathrm{km} 10.7-\mathrm{Gb} / \mathrm{s}$ system with $P_{\mathrm{Tx}}=4 \mathrm{dBm}$ and $\mathrm{OA} 2 \mathrm{Gain}=25 \mathrm{~dB}$.

OA1, which illustrates that the penalty imposed by OA1 is almost equivalent to the noise figure, i.e., the penalty increases on a decibel for decibel basis with the noise figure. Noise figure is defined as a measure of the reduction in SNR imposed by an optical amplifier; hence, this result is sensible. Therefore, it would be sensible to define the noise figure of OA1 to be as low as possible to ensure the best performance. OA2 imposes a much smaller penalty on system performance, as shown in Fig. 4. As the signal at the input of OA1 is free from ASE, the OSNR is defined at this point. The signal and ASE then propagate to the input of OA2, where both are amplified and OA2 adds its internally generated ASE contribution. This contribution is relatively small when compared to the contribution from OA1, which has been amplified by OA2. Therefore, the contribution from OA1 will always have the dominant effect on performance over that of OA2. The penalty imposed by OA3 is greater than OA2 but considerably less than OA1. Hence, in the remaining simulations, the noise figure of OA1 and OA 3 are assumed to be as low as financially possible at $5 \mathrm{~dB}$. Due to the lower penalty OA2 can have a higher noise figure of $6 \mathrm{~dB}$.

3) Optical Amplifier Saturation Power: Simulations were performed, using OptSim, to optimize the saturation power $\mathrm{P}_{\mathrm{SAT}}$ for each optical amplifier in the upstream transmission path of target system, which has a 1024-way-split 100-km reach operating at $10 \mathrm{~Gb} / \mathrm{s}$, with parameters given in Table I, in addition to parameters determined in previous simulations $\left(\alpha_{c 1}=-1.4, \alpha_{c 0}=-1.2, \mathrm{~F}_{\mathrm{OA} 1}=\mathrm{F}_{\mathrm{OA} 3}=5 \mathrm{~dB}\right.$, and $\mathrm{F}_{\mathrm{OA} 2}=$ $6 \mathrm{~dB})$. The system performance is unaffected by the saturation power of OA1, as input-signal power is lower $(-36.3 \mathrm{dBm})$ than that of the output power when the gain of OA1, which is $25 \mathrm{~dB}$, is $-11 \mathrm{dBm}$. Therefore, for significant gain compression to become evident, the saturation power would have to be $<-15 \mathrm{dBm}$. Generally, optical amplifiers have saturation powers which are far greater, and therefore, the saturation power for OA1 is not a critical parameter. The same is true for OA3, which is positioned after a significant amount of attenuation in the form of the backhaul network. The attenuation ensures that the total signal power present at the input to OA3 is low. For

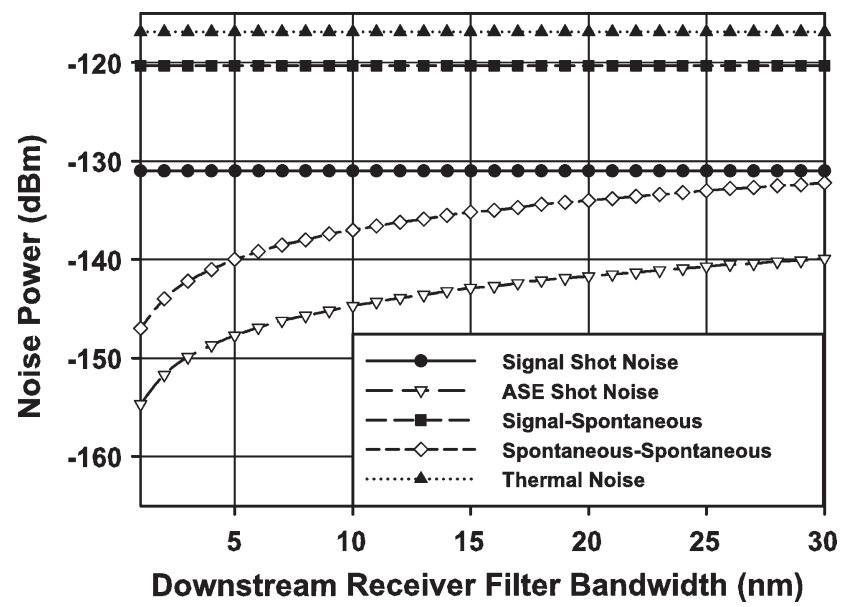

Fig. 6. Noise contribution power at the receiver as the receiver-filter optical-filter bandwidth is varied between 0 and $30 \mathrm{~nm}$. Transmit power $\left(P_{\mathrm{Tx}}=0 \mathrm{dBm}\right)$.

OA2, the simulated system performance shows an increase in performance as the saturation power is increased, as depicted in Fig. 5. System performance increases by $3 \mathrm{~dB}$ as $\mathrm{P}_{\mathrm{SAT}}$ is increased from -5 to $9 \mathrm{dBm}$. Beyond $10 \mathrm{dBm}$, the performance levels off at $Q \sim 11 \mathrm{~dB}$. To maintain the system performance defined by $\mathrm{OA} 1 \mathrm{P}_{\mathrm{SAT}, \mathrm{OA} 2}$ must be greater than $10 \mathrm{dBm}$.

\section{B. Downstream}

As the cost of the OLT is shared among all users, it is possible to place an optical amplifier directly after the transmitter without significantly increasing the cost per user. This is advantageous as the high optical launch power ensures a large OSNR in the downstream path, which enables low BERs to be achieved. Fig. 6 shows the downstream noise modeling performed with the analytical model (transmitter output power, $P_{\mathrm{Tx}}=0 \mathrm{dBm}$ ), illustrating each of the noise contributions as a function of the optical bandwidth at the receiver. Clearly, the thermal noise is the greatest contribution, although the " 1 " signal-spontaneous contribution is only $3 \mathrm{~dB}$ less. Since the system is thermal-noise limited, restricting the optical bandwidth by using optical filters at the receiver will have no effect on the system performance.

The output power of OA3 is a critical parameter in defining the performance of the downstream transmission path. OA3 is situated prior to the distribution section. The total attenuation present in the distribution section, when the split size is 1024 , is $40.3 \mathrm{~dB}$. Such a large amount of attenuation is enough to ensure that ASE power emitted from OA3 is below the receiver noise power, as predicted in Fig. 6. Therefore, to achieve the target performance, the signal power at the receiver must be greater than receiver sensitivity to overcome the thermal noise of the receiver, which defines the system performance in the absence of optical noise. As the sensitivity of the receiver, defined previously in Table $\mathrm{I}$, is $-20 \mathrm{dBm}$ at $\mathrm{BER}=10^{-10}$ to achieve the required performance of $\mathrm{BER}=10^{-10}$, the output signal from OA3 must be greater than $20.3 \mathrm{dBm}$, as shown in the OptSim simulation results for OA3 given in Fig. 7. Such a 


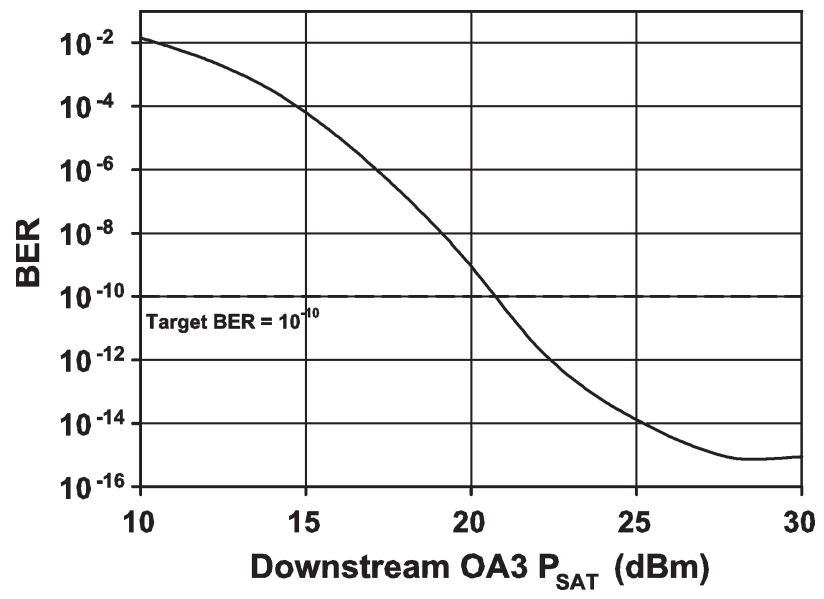

Fig. 7. Downstream system performance when varying $P_{\mathrm{SAT}}$ from 10 to $30 \mathrm{dBm}$ in OA3 for a 1024 -way-split $100-\mathrm{km} 10-\mathrm{Gb} / \mathrm{s}$ system with $P_{\mathrm{Tx}}=0 \mathrm{dBm}$ and $\mathrm{OA} 3 \mathrm{Gain}=25 \mathrm{~dB}$.

large output power does not cause any problems with nonlinear effects as OA3 is immediately followed by the optical splitters, and hence, the optical power is attenuated. Furthermore, nonlinear effects would not be a significant issue, even if the optical splitter were placed farther into the distribution section. This is because the nonlinear length is greater than the distribution section fiber length [16], and the output power level is below the critical power required for stimulated Brillouin scattering [17]. As OA3 is in the shared section of the network, it is not a cost-critical component and will, therefore, have minimal effect on the cost per user.

\section{EXPERIMENTAL SYSTEM}

To prove the feasibility of a long-reach optical-access networks, a laboratory demonstrator based on the system designed in the previous sections was constructed. To enable this, we first focus on the development of the upstream transmission path before moving on to the downstream path.

\section{A. Upstream Demonstrator}

In the experimental setup, as illustrated in Fig. 8, the split loss is simulated by an optical attenuator, which can be adjusted to assess the effect of a varying split size. The attenuator also has a secondary role, which is to pad out the attenuation coefficient of the $10-\mathrm{km}$ SMF fiber reel to $0.35 \mathrm{~dB} / \mathrm{km}$ (fiber-loss coefficient which includes aging and splicing) and simulate additional losses at optical flexible points at the ONU connector and at the input of the local-exchange site. A Northlight Optronics Erbium-Doped Fiber Preamplifier (EDFA) is used for OA1 followed by a Northlight Optronics Booster Amplifier for OA2. In the previous simulations, the optical amplifier gain profile was assumed to be flat. As the gain of practical EDFAs is wavelength-dependent, an ASE filter, with a passband from 1532 to $1565 \mathrm{~nm}$, was placed between OA1 and OA2 in the upstream to remove the 1530-nm gain peak present in the EDFA gain profile. The length of fiber in the backhaul section is 100-km SMF with 18-ps/nm $\cdot \mathrm{km}$ dispersion and a fiber loss co- efficient of $0.35 \mathrm{~dB} / \mathrm{km}$ plus an additional $1 \mathrm{~dB}$ loss at the output of the local-exchange site and $1.5 \mathrm{~dB}$ at the input to the core exchange. A fiber loss coefficient of $0.35 \mathrm{~dB} / \mathrm{km}$ is achieved by adding additional attenuators throughout the backhaul fiber length. The receiver optical amplifier is a JDSU EDFA, which is followed by a receiver with a sensitivity of $-20.75 \mathrm{dBm}$ at $\mathrm{BER}=10^{-10}$ and electrical bandwidth of $12 \mathrm{GHz}$. An optical filter is present before the optical receiver, which is to be changed in order to adjust the optical bandwidth of the system. An 18-nm thin-film filter was available for use, but the other bandwidths were realized by using the filter mode of an Agilent 86146B OSA. The transmitter used is an XFP MSA-compliant TXN18117 transceiver and is manufactured by Intel. It is designed to support bit rates from 9.95 to $10.71 \mathrm{~Gb} / \mathrm{s}$ and is integrated with clock and data recovery circuits designed to support optical links from 2 to $40 \mathrm{~km}$. To make the device suitable for the long-reach optical-access networks application, it was tuned through simple adjustment of the electrical parameters (voltage swing, crossing point, voltage offset, and temperature control) to allow transmission over greater distances. The transmitted nonreturn to zero intensity-modulated signal has an extinction ratio of approximately $9 \mathrm{~dB}$ with $4-\mathrm{dBm}$ launch power. To assess the performance of the upstream transmission path, an optical attenuator was used to simulate a varying split size. The BER of the system was measured at $1-\mathrm{dB}$ intervals as the total loss of the distribution section was varied from 42.3 to $31.3 \mathrm{~dB}$. Using this method, the power into OA1 is increased, ultimately resulting in an increasing OSNR at the receiver as the split size is decreased. Increasing the input power to OA1 increases the system performance as shown in the BER performance curves in Fig. 9, which also shows the effect of changing the optical bandwidth. Reducing the receiver-filter bandwidth in the upstream transmission path of the long-reach optical-access networks increases the system performance. Specifically, a 2-nm optical receiver filter improves system performance by $2 \mathrm{~dB}$ in comparison with a receiver filter with an optical bandwidth of $18 \mathrm{~nm}$. Performance of the system with a split size of 1024 and a 2 -nm optical bandwidth is BER $\sim 2 \times 10^{-3}$ above the pre-FEC BER $=2.9 \times 10^{-4}$ required to achieve target performance of BER $=10^{-10}$. Specifying an ONU source with 2-nm accuracy is a made feasible through the use of an MSA-compliant XFP transceiver-cooled transmitter. The device should be extremely cost effective through volume production, as it is suitable for a number of transmission formats including 10G Ethernet. Hence, a 2-nm source can be specified without a significant increase in the ONU cost. Performance of the system could also be increased by reducing the split size to 512 , which would give $\mathrm{BER}=1.5 \times 10^{-5}$, which is below the required target for operation with FEC. Operation without FEC requires the distribution section loss to be reduced to $28 \mathrm{~dB}$, corresponding to a split of $96-$ and $100-\mathrm{km}$ reach. A method of improving the upstream performance by reducing the dispersion penalty exists in the form of electronic dispersion compensation (EDC). EDC consists of an adaptive electronic filter, which is placed at the receiving end of the system. This is beneficial for long-reach optical-access networks, since EDC will only be placed at the OLT, and hence, the cost can be shared across all users. 


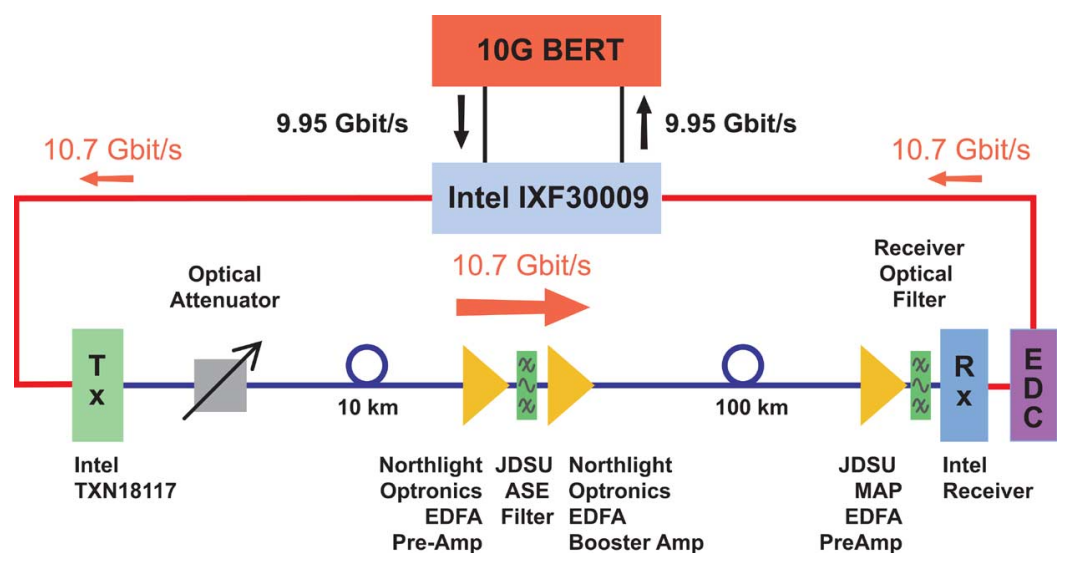

Fig. 8. Upstream experimental configuration.

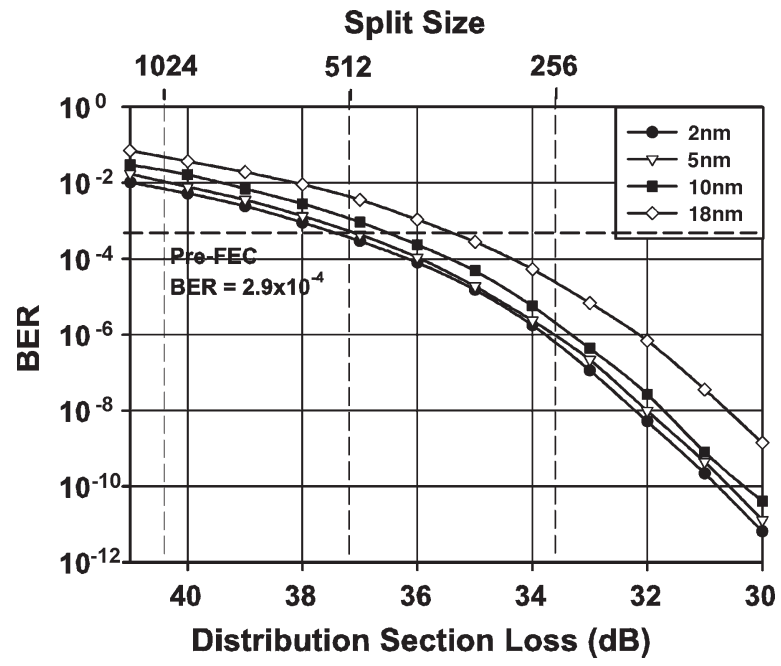

Fig. 9. Experimental BER using an Intel TXN18117 transmitter at optical bandwidths of $2,5,10$, and $18 \mathrm{~nm}$.

The EDC circuit used was supplied by Intel and consists of a finite-impulse-response filter. System performance of the upstream path with 2-nm optical bandwidth can be improved further by combining EDC and FEC, as shown in Fig. 10. When using FEC with the EDC and a 2-nm bandwidth, it is possible to support a split size of 930 with BER $=10^{-10}$, which falls short of the goal of a 1024-way split. Using FEC is feasible, if the split size is limited to 512 , which would be more practical as it would allow the system to operate with considerable margin. The increased gain available with SuperFEC allows a split of 1300 to be supported. This is greater than the 1024 split required, and hence, a 1024-way-split 100-km 10-Gb/s systems would be able to operate with some performance margin.

\section{B. Downstream Demonstrator}

The downstream experimental configuration is illustrated in Fig. 11. Increased supported split size as a result of combining FEC and SuperFEC with EDC and a 2-nm optical bandwidth is shown in Fig. 10.

As with the upstream setup, optical attenuators are used to simulate the optical couplers to pad out the single-mode fiber

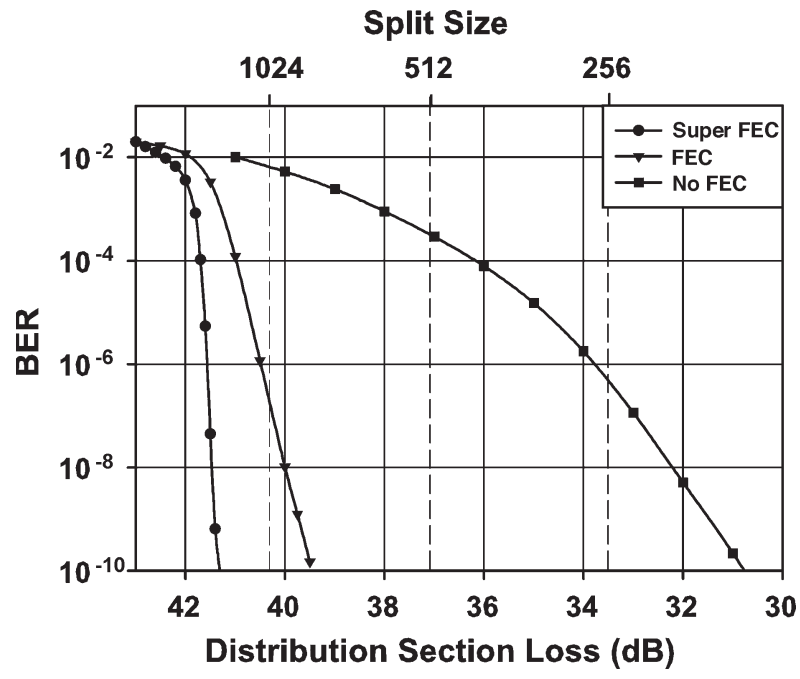

Fig. 10. Increased supported split size as a result of combining FEC and SuperFEC with EDC and a 2-nm optical bandwidth.

loss to $0.35 \mathrm{~dB} / \mathrm{km}(D=18 \mathrm{ps} / \mathrm{nm} \cdot \mathrm{km})$ and to add additional loss at the flexibility points in both the distribution and backhaul sections. A high-power Bookham MGMFL-2AUC28 EDFA is used for OA3 to ensure that the input signal to the distribution section is greater than $20 \mathrm{dBm}$. For OA1 and OA2, a Northlight Optronics Preamplifier and JDSU Booster EDFA are used, respectively. The receiver sensitivity is approximately $-20 \mathrm{dBm}$ at $\mathrm{BER}=10^{-10}$.

Downstream transmission is considerably better than upstream. Fig. 12 shows that the Intel TXN13600 is capable of the target performance: $\mathrm{BER}=10^{-10}$ with a 1024 -split $100-\mathrm{km}$ reach at $10 \mathrm{~Gb} / \mathrm{s}$ without FEC. FEC could be used to reduce OA3's output power. Upstream, EDC was used to counter the dispersion penalty of the most cost-effective upstream transmitter. EDC and FEC in the downstream could be considered but would require a more complex ONU, which should be avoided on economic grounds.

Another method of dispersion management is to use dispersion-compensating fiber (DCF). Downstream performance analysis was performed with DCF. The performance of the Intel TXN13600 downstream DCF system is given in 


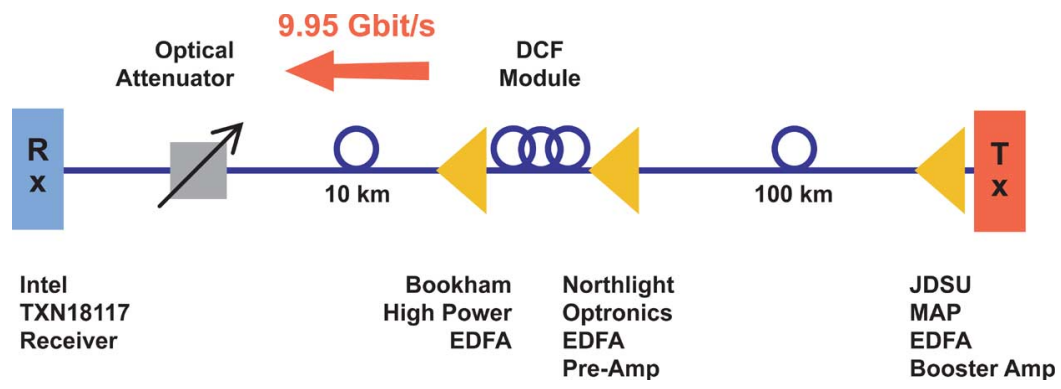

Fig. 11. Downstream experimental configuration.

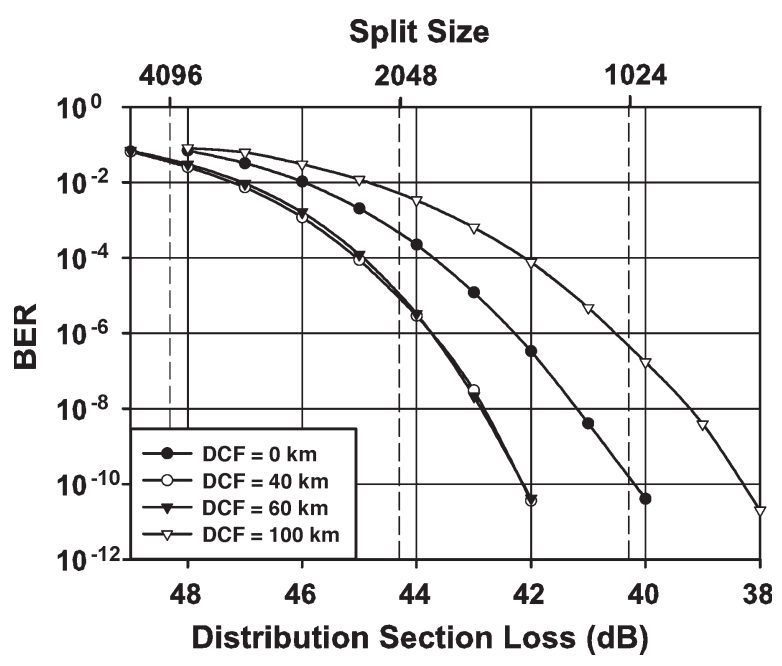

Fig. 12. Downstream experimental results using Intel TXN13600 transmitter and DCF.

Fig. 12. Performance of the 40- and 60-km DCF systems is almost identical, demonstrating that the dispersion penalties at 60 and $40 \mathrm{~km}$ of SMF fiber are equal. The split size increase over the case with no DCF is 1650 . Increasing the DCF compensation length to $100 \mathrm{~km}$ is detrimental to system performance due to the interaction of chirp and dispersion. The dispersion penalty is increased over the case with no DCF. Hence, $100 \mathrm{~km}$ of DCF reduces the split size supported to 700 at the target performance.

\section{CONClusion}

This paper has provided a summary of our work, which proves the feasibility of 1024-way-split $100-\mathrm{km} 10-\mathrm{Gb} / \mathrm{s}$ longreach optical-access networks. In the upstream transmission path, SuperFEC and EDC are required in addition to a chirp-assisted transmitter to achieve the target performance of $\mathrm{BER}=10^{-10}$. Downstream, target performance can be achieved without FEC or EDC, provided that an EDFA with $P_{\mathrm{SAT}}>21 \mathrm{dBm}$ is used prior to the distribution section. The downstream performance can be improved by introducing DCF, which optimizes the dispersion penalty. Therefore, this paper has provided comprehensive proof that long-reach optical-access networks are feasible both economically and technically. However, further work remains to study any impairment, which may be introduced through burst-mode operation.

\section{ACKNOWLEDGMENT}

The authors would like to thank R. Davey and D. Nesset of BT for their support of this work and access to the facilities at Adastral Park.

\section{REFERENCES}

[1] ITU-T Recommendation G.983.1: 'Broadband Optical Access Systems Based on Passive Optical Networks (PON),' 1998, Geneva, Switzerland: Int. Telecommun. Union.

[2] ITU-T Recommendation G.984.1: 'Gigabit-Capable Passive Optical Networks (GPON): General Characteristics,' 2003, Geneva, Switzerland: Int. Telecommun. Union.

[3] I. G. Dufour, "Local lines-The way ahead," Br. Telecommun. Eng., vol. 4 , pt. 1 , pp. $47-51,1985$.

[4] I. Hawker, S. Whitt, and G. R. Bennett, "The future British Telecom core transmission network," in Proc. 2nd IEE Nat. Conf. Telecommun., 1989, p. 364.

[5] J. Vandenbroeck, FIST-SASA2 Ordering Guide. Harrisburg, PA: Tyco/ Electronics/Raychem, Jan. 2000.

[6] Y. K. Park and S. W. Granlund, "Optical preamplifier receivers: Application to long-haul digital transmission," Opt. Fiber Technol., vol. 1, no. 1, p. 59 , Oct. 1994.

[7] D. P. Shea, R. P. Davey, A. Lord, and J. E. Mitchell, "Design aspects of long reach optical access networks," presented at the Int. Symp. Services and Local Access, Edinburgh, U.K., 2004.

[8] T. L. Koch, "Laser sources for amplified and WDM systems," Optical Fibre Communications IIIB, pp. 115-163, 1997.

[9] ITU-T Recommendation G.975: 'Forward Error Correction for Submarine Systems', 2000, Geneva, Switzerland: Int. Telecommun. Union.

[10] G. P. Agrawal and M. J. Potasek, "Effect of frequency chirping on the performance of optical communication systems," Opt. Lett., vol. 11, no. 5, pp. 318-320, May 1986.

[11] D. G. Moodie, A. D. Ellis, P. J. Cannard, C. W. Ford, A. H. Barrell, R. T. Moore, S. D. Perrin, R. I. McLaughlin, and F. C. Garcia, " $40 \mathrm{~Gb} / \mathrm{s}$ modulator with low drive voltage and high optical output power," in Proc. ECOC, Amsterdam, The Netherlands, 2001, pp. 332-333.

[12] T. Mizuochi, K. Kubo, H. Yoshida, H. Fujita, H. Tagami, M. Akita, and K. Motoshima, "Next generation FEC for optical transmission systems," in Proc. OFC, 2003, pp. 527-528.

[13] S. Keeton, S. Sridharan, and M. Jarchi, "Enabling next generation optical networks with forward error correction," in Proc. NFOEC, 2001, vol. 1, pp. 54-59.

[14] H. Stiles and T. Wolcott, "Avantages of RF communication technologies in optical subcarrier multiplexed systems," in Proc. NFOEC, 2001, vol. 2, pp. 1025-1034.

[15] G.709: The Optical Transport Network (OTN), 2002, Acterna Whitepaper. [Online]. Available: http://www.acterna.com/global/technical_resources/ white_papers/\#O

[16] P. Bayvel and R. Killey, "Nonlinear optical effects in WDM transmission," in Optical Fibre Telecommunications. New York: Academic, 2002, pp. 611-641.

[17] R. G. Smith, "Optical power handling capacity of low loss optical fibres as determined by stimulated Raman and Brillouin scattering," J. Appl. Opt., vol. 11, no. 11, pp. 2489-2494, 1972. 


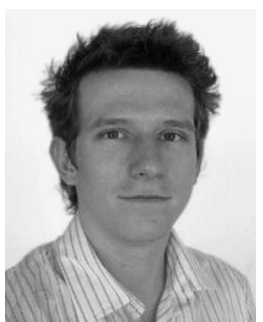

Darren P. Shea received the degree (with firstclass honors) in electronic engineering from The University of Manchester, Manchester, U.K., and the Eng.D. degree in telecommunications from University College London (UCL), London, U.K., in 2006. His thesis research, performed in conjunction with BT, involved the design and construction of a $10-\mathrm{Gb} / \mathrm{s}$ optical-access network, which connected 1024 customers directly to the core network over a distance of $100 \mathrm{~km}$.

For the duration of his doctorate research, he worked as part of the Optical Networks group at BT, Adastral Park, Ipswich, U.K. He is currently working as a Research Fellow with the UCL. His research interests include optical access, specifically fiber to the home, and burst-mode receiver and long-haul transmission improvements using optical frequency chirping.

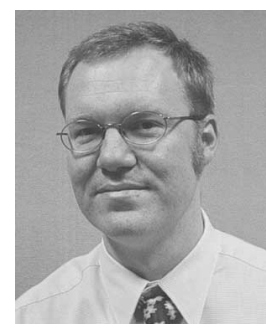

John E. Mitchell (S'97-M'00) received the B.Eng. and Ph.D. degrees in electronic and electrical engineering from University College London (UCL), London, U.K., in 1996 and 2000, respectively. His thesis work dealt with interferometric noise and related phenomena in optical networks.

In 1999, he became a research fellow with the Department of Electronic and Electrical Engineering, UCL, becoming a Lecturer and Senior Lecturer in telecommunications, in 2000 and 2006, respectively. He has published over 40 papers in the area of optical communications. His research interests include optical-access technologies and the transport of millimeter wave radio signals over the optical fiber for communications and noise relating to crosstalk in optical networks.

Dr. Mitchell is a member of the Institute of Electronic Engineers and the IEEE Leasers and Electro-Optics Society. 